\title{
Darwin Cloud: Mapping real-world data to Darwin
}

\section{Core}

John Wieczorek ${ }^{\ddagger} \S$, Paul J. Morris§, James Hanken§, David B. Lowery§, Bertram Ludäscherl, James Macklin $\llbracket$, Timothy McPhillips!, Robert A. Morris \#,§, Qian Zhangl

‡ Museum of Vertebrate Zoology, University of California, Berkeley, United States of America

$\S$ Museum of Comparative Zoology, Harvard University, Cambridge, MA, United States of America

| University of Illinois Urbana-Champaign, Champaign, United States of America

I Agriculture and Agri-Food Canada, Ottawa, Canada

\# University of Massachusetts, Boston, Boston, United States of America

Corresponding author: John Wieczorek (tuco@berkeley.edu), Paul J. Morris (씽@orris.net)

Received: 21 Aug 2017| Published: 21 Aug 2017

Citation: Wieczorek J, Morris P, Hanken J, Lowery D, Ludäscher B, Macklin J, McPhillips T, Morris R, Zhang Q

(2017) Darwin Cloud: Mapping real-world data to Darwin Core. Proceedings of TDWG 1: e20486. https://doi.org/10.3897/tdwgproceedings.1.20486

\section{Abstract}

Since its ratification as a TDWG standard in 2009, data publishers have had to struggle with the essential step of mapping fields in working databases to the terms in Darwin Core Wieczorek et al. 2012 in order to publish and share data using that standard. Doing so requires a good understanding of both the data set and Darwin Core. The accumulated knowledge about these mappings constitutes what we call the "Darwin Cloud." We will explore the nature of data mapping challenges and the potential for semi-automated solutions to them. Specifically, we will look at the "Darwinizer" actor and its usage in related workflows within the Kurator data quality framework and the implications for communitymanaged vocabularies.

\section{Keywords}

Biodiversity Informatics, Darwin Core, Data Quality 


\section{Presenting author}

John Wieczorek

\section{Funding program}

NSF DBI 1356438 and 1356751

\section{References}

- Wieczorek J, Bloom D, Guralnick R, Blum S, Döring M, Giovanni R, Robertson T, Vieglais D (2012) Darwin Core: An Evolving Community-Developed Biodiversity Data Standard. PLoS ONE 7 (1): e29715. https://doi.org/10.1371/journal.pone.0029715 